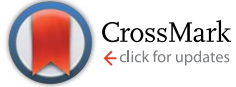

Cite this: J. Mater. Chem. A, 2016, 4, 13211

Received 6th July 2016

Accepted 29th July 2016

DOI: 10.1039/c6ta05661j

www.rsc.org/MaterialsA

\section{Elevated salt transport of antimicrobial loose nanofiltration membranes enabled by copper nanoparticles via fast bioinspired deposition $\dagger$}

\author{
Junyong Zhu, ${ }^{a}$ Adam Uliana, ${ }^{\text {ab }}$ Jing Wang, ${ }^{\text {ac }}$ Shushan Yuan, ${ }^{a}$ Jian Li, ${ }^{a}$ Miaomiao Tian, ${ }^{c}$ \\ Kenneth Simoens, ${ }^{a}$ Alexander Volodin, ${ }^{d}$ Jiuyang Lin, ${ }^{* e}$ Kristel Bernaerts, ${ }^{a}$ \\ Yatao Zhang ${ }^{\mathrm{C}}$ and Bart Van der Bruggen ${ }^{\star a}$
}

Surface functionalization with advanced nanomaterials offers tailored control and targeted design of surface properties, endowing materials with enhanced or new qualities such as high hydrophilicity, excellent selectivity and permeability, and enhanced antimicrobial activity. In this study, we develop two strategies (two-step deposition/co-deposition) that use mussel-inspired polydopamine (PDA) to strongly immobilize copper nanoparticles (CUNPs) onto a porous polymeric membrane, bridging the surface cavities from ultrafiltration (UF) to loose nanofiltration (NF). To confirm the optimization of membrane properties, a series of characterizations were carried out: SEM, EDX analysis, AFM, water contact angle, and zeta potential measurements. The results indicate an overall high performance of surface properties with a homogeneous nanoparticle distribution, low roughness, favorable hydrophilicity, and relatively neutral charge. Co-deposition of PDA and CuNPs exhibits a facile and time-saving process that expedited a higher CUNP loading compared to the two-step strategy, as confirmed by SEM and AFM images. The integration of polyethylenimine (PEI)-modified CUNPs with high density of positive charges plays an important role in fine-tuning the hydrophilicity and compatibility with PDA and in largely neutralizing the negative charge of PDA, thus promoting an outstanding salt permeation $\left(82 \% \mathrm{Na}_{2} \mathrm{SO}_{4}\right.$, $98 \% \mathrm{NaCl}$ ). In addition, CuNP/PDA-modified membranes show an ultra-high rejection of three types of textile dyes (600-800 Da, >99.0\%), demonstrating superior NF performance. Furthermore, the functionalized membranes display distinct bactericidal activity with a great reduction of $93.7 \%$ in the number of live Escherichia coli (E. coli) bacteria. This study highlights a fast, facile co-deposition strategy to assemble multifunctional coating onto a UF support, which renders a vast potential for the fractionation of dye/salt mixtures.

\section{Introduction}

Nanofiltration (NF) is one of the most viable water treatment solutions to the current worldwide water crisis. In textile wastewater treatment, NF technology is increasingly becoming applied in a new paradigm shift to separate, recycle, and reuse

\footnotetext{
${ }^{a}$ Department of Chemical Engineering, KU Leuven, Celestijnenlaan 200F, B-3001 Leuven, Belgium. E-mail: bart.vanderbruggen@kuleuven.be

${ }^{b}$ Department of Chemical Engineering, The Pennsylvania State University, University Park, PA 16802, USA

${ }^{c}$ School of Chemical Engineering and Energy, Zhengzhou University, Zhengzhou 450001, China

${ }^{d}$ Laboratory of Solid-State Physics and Magnetism, Department of Physics and Astronomy, KU Leuven, Celestijnenlaan 200D, B-3001 Leuven, Belgium

eSchool of Environment and Resources, Qi Shan Campus, Fuzhou University, No. 2 Xueyuan Road, University Town, 350116 Fuzhou, Fujian, China. E-mail: linjiuyang@126.com

$\dagger$ Electronic supplementary information (ESI) available. See DOI: 10.1039/c6ta05661j
}

dye/salt mixtures as resources rather than remove them completely as waste. ${ }^{1-4}$ Commercially available NF membranes generally consist of a thin compact polyamide layer constructed on a microporous support and have a relatively high retention of divalent salts $\left(\mathrm{Na}_{2} \mathrm{SO}_{4}\right.$ : $\left.>90 \%\right) .{ }^{5}$ Such dense membranes are committed to water desalination and consequently fail in sufficient separation of dye/salt mixtures. Moreover, low salt permeation through membranes can pose a high osmotic pressure, further compromising their water permeability and inevitably impeding potential NF applications. ${ }^{6}$

Conversely, loose NF membranes, which are properly tuned to have a loose separating layer and controlled surface charges, feature a high water flux and superior dye retention combined with an enhanced salt permeation. ${ }^{7-9}$ So far, loose NF membranes have emerged as an effective remedy for fractionating dye/salt mixtures. ${ }^{10,11}$ Currently, they have been constructed by blending a flexible polymer with functional nanoparticles, including graphene oxide, ${ }^{2}$ montmorillonite, ${ }^{8}$ $\mathrm{SiO}_{2},{ }^{12}$ layered double hydroxides, ${ }^{13}$ and halloysite nanotubes, ${ }^{\mathbf{1 4}}$ 
followed by a phase inversion process. Such a technique substantially boosts the water flux, salt permeation, and antifouling properties of the membranes. These enhanced abilities are presumably attributed to the introduced combination of high hydrophilicity, loose structure, and controllable charge of the membrane surface. Nonetheless, this strategy is based on partially sacrificing dye rejection due to the relatively poor compatibility between hard inorganic nanoparticles and the soft polymeric matrix. ${ }^{15}$ Furthermore, nanocomposites embedded inside the membrane matrix are also not directly exposed to the solution being treated, minimizing the functionality effects of the nanocomposites. ${ }^{\mathbf{1 6}}$

Surface modification of polymeric membranes is deemed a simple and effective strategy. It enables tuneable and controllable surface properties that enhance permeability, selectivity, and antifouling ability when being implemented for water treatment. The most extensively employed surface functionalization methods involve interfacial polymerization, ${ }^{\mathbf{1 7 - 2 0}}$ coating, ${ }^{21}$ surface grafting, ${ }^{22}$ covalent immobilization, ${ }^{23}$ layer-bylayer assembly, ${ }^{24}$ crosslinking, ${ }^{24}$ etc. Unfortunately, these methods frequently involve tedious, lengthy, and complicated steps that can also indirectly harm the environment. Notably, the formation of a compact active layer using the aforementioned strategies is unsuitable for dye/salt separation.

Mussel-inspired polydopamine (PDA) has been explored as an alternative surface modification approach by numerous studies since $2007 .{ }^{25}$ PDA possesses inherently strong adhesive properties to a vast variety of substrates due to the presence of multifunctional groups (amine, catechol, and imine). ${ }^{26,27}$ Therefore, PDA can act as a sturdy bioinspired glue to firmly immobilize favorable nanomaterials onto membrane surfaces without any damage. Membranes modified with PDA have shown large advances in hydrophilicity, permeability, and antifouling performance. ${ }^{28-30} \mathrm{Ag},{ }^{31} \mathrm{TiO}_{2},{ }^{32-34} \mathrm{SiO}_{2},{ }^{35}$ and $\mathrm{ZrO}_{2}$ (ref. 36) inorganic nanoparticles have been successfully loaded onto membrane surfaces using the self-polymerization and deposition of dopamine. With these surface functionalization capabilities, PDA has stimulated extensive interest in the design of water treatment membranes, such as microfiltration (MF), ${ }^{37}$ ultrafiltration (UF), ${ }^{38} \mathrm{NF},{ }^{39,40}$ reverse osmosis (RO), ${ }^{41}$ and forward osmosis (FO) ${ }^{42}$ membranes. However, its self-polymerization and deposition onto membrane surfaces is a timeconsuming process and simultaneously leads to inevitable aggregation. ${ }^{43}$ Despite the existence of such difficulties, Zhang et al. reported the first procedure to achieve the rapid deposition of PDA onto various substrates by utilizing $\mathrm{CuSO}_{4} / \mathrm{H}_{2} \mathrm{O}_{2}$ as a trigger. ${ }^{44} \mathrm{CuSO}_{4} / \mathrm{H}_{2} \mathrm{O}_{2}$ generates a multitude of reactive oxygen species (ROS) in an alkaline medium to significantly expedite the deposition rate of PDA coatings with high uniformity and stability, vastly expanding the potential of PDA in practical applications.

Membrane biofouling is another major obstacle to filtration processes. It sharply increases energy consumption and reduces the filtration productivity. Biofouling originates from the adsorption of biofilms initiated by rapid bacterial reproduction and proliferation. Bacteria from the adsorbed biofilm can redisperse into the aqueous environment, polluting the treated solution. Biofilms are typically embedded in a naturally formed matrix of extracellular polymeric substances (EPS), which contain polysaccharides, proteins, glycoproteins, lipoproteins, and other bio-macromolecules. ${ }^{45}$ The EPS can provide protection to the biofilm against biocides, rendering the killing of microorganisms more difficult. Therefore, the most effective route toward long-term resistance against biofouling is to inhibit the initial cell adhesion and growth onto membrane surfaces. Anchoring antimicrobial polymers or nanoparticles onto membrane surfaces allows these nanomaterials to maintain maximum contact with natural organic matter, proteins, and bacteria to reduce their adherence and growth. ${ }^{46-48}$

One of the most studied antimicrobial nanomaterials consists of silver-based nanocomposites owing to their thermal stability and broad antibacterial spectrum. ${ }^{49,50}$ However, silverbased biocides carry large financial costs, raising concerns about a sustainable design of antimicrobial membranes. A growing research focus has shifted toward the use of copperbased nanoparticles as biocides instead to strengthen the surface resistance to biofouling. ${ }^{51,52}$ While the antimicrobial properties of copper have been widely established in the literature, copper is also rather cheap ( $\sim 1 \%$ of the price of silver) and abundant. ${ }^{53}$ Ben-Sasson et al. reported a simple technique to load positively charged CuNPs modified by polyethylenimine (PEI) onto the surface of a thin-film composite polyamide RO membrane via electrostatic interaction. ${ }^{54}$ This surface functionalization led to a remarkable gain of bacterial inactivation. Their group also recently presented in situ growth of CuNPs onto membrane surfaces as an alternative functionalization method against biofouling. ${ }^{55}$ Likewise, $\mathrm{Xu}$ et al. developed a simple assembly of a polycation-copper(II) complex onto a polyacrylonitrile (PAN) substrate followed by crosslinking to prepare a CuNP-functionalized antibacterial NF membrane. ${ }^{56}$ Nevertheless, the depletion rate of copper ions is higher than copper nanoparticles. These membranes also had a high rejection of bivalent salts, which is ill-suited for the separation of dye/salt mixtures.

In this study, we introduce and compare two simple methods to assemble a multifunctional CuNP layer onto a porous polymeric support by applying the fast deposition of PDA. One of these techniques refers to a conventional two-step deposition by the aid of a strongly adhesive PDA intermediate layer followed by CuNP loading. The second strategy is the simultaneous codeposition of PDA and CuNPs. These two strategies are compared and studied in view of surface morphology, solute selectivity, and permeability. The antimicrobial activity of the co-deposition membranes is further studied by using E. coli as model bacteria.

\section{Experimental}

\subsection{Chemicals and materials}

Copper(II) sulfate $\left(\mathrm{CuSO}_{4}, 99 \%\right)$, hydrogen peroxide $\left(\mathrm{H}_{2} \mathrm{O}_{2}, 30 \%\right.$ by weight), sodium borohydride $\left(\mathrm{NaBH}_{4}, 98 \%\right)$, polyethylenimine (PEI, branched, $\mathrm{MW}=25 \mathrm{kDa}$ ), hydrochloric acid ( $\mathrm{HCl}, 1 \mathrm{M}$ ), sodium hydroxide ( $\mathrm{NaOH}, 98 \%)$, tris(hydroxymethyl) aminomethane (Tris, 99.8\%), and dopamine hydrochloride 
were acquired from Sigma-Aldrich (Diegem, Belgium). Polyacrylonitrile UF sheet membranes (PAN, MWCO = $75 \mathrm{kDa}$ ) were kindly supplied by Ultura (USA). Direct red 23 (DR23, dye content: $>30 \%$, Sigma-Aldrich), Congo red (CR, pure, Avocado Research Chemicals Ltd, United Kingdom), and reactive blue 2 (RB2, pure, Sigma-Aldrich) were chosen as model dyes tested at the concentration of $0.5 \mathrm{~g} \mathrm{~L}^{-1}$. Details of the dyes are listed in Table 1. Magnesium chloride $\left(\mathrm{MgCl}_{2}, 98 \%\right)$, sodium chloride $(\mathrm{NaCl}, 99 \%)$, and sodium sulfate $\left(\mathrm{Na}_{2} \mathrm{SO}_{4}, 99 \%\right)$ were purchased from Sigma-Aldrich and were tested for salt permeation. Unless specified, all solutions were prepared in deionized (DI) water purified by a Milli-Q ultrapure unit.

\subsection{Membrane modifications}

Prior to surface modifications, the PAN membranes were hydrolyzed via immersion into $\mathrm{NaOH}$ aqueous solution $(2 \mathrm{M})$ at ambient temperature for $20 \mathrm{~h}$. The membranes were subsequently soaked in DI water for $24 \mathrm{~h}$ to create hydrolyzed PAN (HPAN) membranes. All the membranes used in this study were stored in DI water until use.

2.2.1. Synthesis of PEI-modified CuNPs. A PEI-modified CuNP suspension was synthesized using $\mathrm{NaBH}_{4}$ as a reductant based on an improved method. ${ }^{54} \mathrm{PEI}$ regarded as a polycationic capping agent can effectively restrict particle aggregation. $\mathrm{CuSO}_{4}$ solution $(50 \mathrm{mM})$ was mixed with PEI solution (0.066 $\mathrm{mM}$ ) while stirring at $\sim 300 \mathrm{rpm}$ for $5 \mathrm{~min}$. The reduction of copper ions into CuNPs was initiated by adding $\mathrm{NaBH}_{4}$ solution $\left(V_{\mathrm{CuSO}_{4}}: V_{\mathrm{PEI}}: V_{\mathrm{NaBH}_{4}}=1: 3: 1\right.$, where $V$ denotes solution volume) into the mixture while stirring at $\sim 300 \mathrm{rpm}$ for $20 \mathrm{~min}$. Afterwards, $\mathrm{H}_{2} \mathrm{O}_{2}\left(V_{\mathrm{H}_{2} \mathrm{O}_{2}} / V_{\text {mixture }}=0.8 \%\right)$ was added, changing the solution color to dark green. Finally, the ensuing CuNP suspension was sonicated (Transsonic T780, Elma) for $20 \mathrm{~min}$ before use.

2.2.2. Preparation of PDA-modified (PDA-HPAN) membranes. $\mathrm{CuSO}_{4}(5 \mathrm{mM})$ was first dissolved in Tris buffer solution $(50 \mathrm{mM}, \mathrm{pH}=8.5) . \mathrm{H}_{2} \mathrm{O}_{2}(19.6 \mathrm{mM})$ was then mixed into the solution, which was followed by the addition of dopamine hydrochloride $\left(2 \mathrm{mg} \mathrm{mL} \mathrm{m}^{-1}\right)$. The resulting dopamine solution $(40 \mathrm{~mL})$ was poured into a membrane holder where only the surface of the dried HPAN membrane can come into contact with the solution for different times $(20 \mathrm{~min}, 30 \mathrm{~min}$, and $6 \mathrm{~h}$ ) at a static state following the strategy proposed by Zhang et al., ${ }^{\mathbf{4 4}}$ enabling the rapid self-polymerization and uniform deposition of PDA. Herein, for surface modification, the digital photos of membrane holders for fixing the membranes are shown in Fig. S1. $\dagger$ Membranes were rinsed for five times and submerged in DI water overnight.

2.2.3. Deposition of CuNPs onto HPAN and PDA-HPAN membranes. Fig. 1 presents the schematic process of surface functionalization by PDA and CuNPs. To fabricate the two-step deposition membranes, the dried PDA-HPAN membrane surface was covered by the synthesized CuNP suspension (40 $\mathrm{mL}$ ) for different times $(6,12,18$, and $24 \mathrm{~h})$. The CuNP suspension $(40 \mathrm{~mL})$ was also deposited onto the dried HPAN membrane to develop a CuNP-functionalized membrane without PDA for comparison. Membranes were rinsed five times and submerged in DI water overnight.

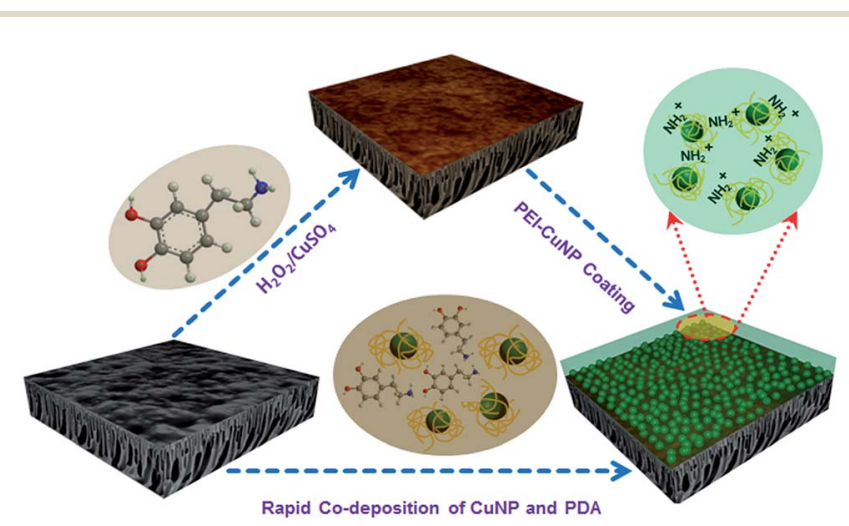

Fig. 1 Schematic diagram of the surface modifications of the HPAN membrane via two-step deposition and co-deposition by using PDA and CuNPs.

Table 1 Characterizations of direct and reactive dyes used in this study

Direct red $23(\mathrm{DR} 23)$

${ }^{a}$ Molecular weight. ${ }^{b}$ Maximum absorption wavelength. 
2.2.4. Co-deposition of PDA and CuNPs onto HPAN membranes. Dopamine solution $(25 \mathrm{~mL})$ was prepared as described in the preparation of PDA-HPAN; then it was poured on top of the HPAN membrane. After $10 \mathrm{~s}$, CuNP suspension $(25 \mathrm{~mL})$ was introduced into the dopamine solution, which was then shaken for $5 \mathrm{~min}$. Membranes were left at a static state for functionalization for different times $(1.5,3,6,12$, and $24 \mathrm{~h})$. The samples were then rinsed five times and submerged in DI water overnight. The functionalization parameters and assigned labels of the modified membranes used in this study are summarized in Table S1. $\dagger$

\subsection{Membrane characterization}

2.3.1. Scanning electron microscopy (SEM). The surface morphologies of the membranes were visualized by SEM imaging via a Philips Scanning Electron Microscope XL30 FEG (Netherlands) with an energy dispersive X-ray (EDX) detector, which was also used to perform energy dispersive X-ray spectroscopy (EDS) compositional analysis. Each sample was dried before analysis in a vacuum chamber and then sputter-coated with gold.

2.3.2. Atomic force microscopy (AFM). AFM measurements were performed to determine the surface roughness of the membranes. AFM measurements were taken under ambient conditions using a Dimension 3100 AFM device (Bruker, USA) with scan areas of $1 \times 1 \mu^{2}$. AFM images were taken in tapping mode using NCSHR probes (NanoAndMore GmbH, Germany). The cantilever was composed of Si with a spring constant of 40$50 \mathrm{~N} \mathrm{~m}^{-1}$ and a nominal tip apex radius of $<7 \mathrm{~nm}$. The AFM images were flattened with order 1 after scanning to remove slope and curvature from images. Root-mean-squared roughness $\left(R_{\mathrm{rms}}\right)$ values were calculated after flattening as explained in the previous study. ${ }^{57}$ Additionally, the average roughness $\left(R_{\mathrm{a}}\right)$ and maximum vertical distance between the highest and lowest points $\left(R_{\mathrm{m}}\right)$ of the membrane surfaces were also investigated.

2.3.3. Water contact angle. Water contact angle measurements were conducted to study the hydrophilicity of the membranes. A contact angle goniometer (OCA20, Dataphysics Instruments, Germany) was operated at room temperature with $40 \%$ relative humidity. DI water $(2 \mu \mathrm{L})$ was dropped onto the membrane surface for each measurement. Reported contact angles represent the recorded average of five randomly selected locations on each sample.

2.3.4. Surface charge. Zeta potential was tested via streaming potential measurements on an electrokinetic analyzer (SurPASS Anton Paar, Austria). Flat membrane samples $(0.2 \mathrm{~cm} \times 0.1 \mathrm{~cm})$ were mounted onto the adjustable gap cell. $\mathrm{KCl}$ solution $(1.0 \mathrm{mM})$ was chosen as the background solution. $\mathrm{HCl}(0.1 \mathrm{M})$ and $\mathrm{KOH}(0.1 \mathrm{M})$ were applied to adjust the $\mathrm{pH}$ over a range of 3-11. Measurements were determined using the Visolab for SurPASS software.

2.3.5. Filtration performance tests. Water flux, salt retention, and dye retention were tested with the lab-made cross-flow filtration setup described in previous studies to evaluate membrane filtration performance. ${ }^{1}$ Membrane coupons with an effective area of $22.9 \mathrm{~cm}^{2}$ were pre-pressurized with DI water for
$30 \mathrm{~min}$ at 8 bar to reach a steady state before testing. After the compaction, the water fluxes were recorded at four different pressures ranging from 1 to 8 bar; the average of the four values was reported. Salt $\left(\mathrm{MgCl}_{2}, \mathrm{NaCl}\right.$, and $\left.\mathrm{Na}_{2} \mathrm{SO}_{4}, 1 \mathrm{~g} \mathrm{~L}{ }^{-1}\right)$ permeation was measured at 6 bar with a flow velocity of $30 \mathrm{~L} \mathrm{~h}^{-1}$. In addition, dye (DR23, CR, and $\mathrm{RB} 2,0.5 \mathrm{~g} \mathrm{~L}^{-1}$ ) retention was separately measured at 8 bar with a flow velocity of $20 \mathrm{~L} \mathrm{~h}^{-1}$. The permeation flux $(J)$, water permeability (WP), and retention $(R)$ were calculated using the following equations:

$$
\begin{gathered}
J=\frac{V}{A \times \Delta t} \\
\mathrm{WP}=\frac{J}{\Delta P} \\
R=\frac{C_{\mathrm{f}}-C_{\mathrm{p}}}{C_{\mathrm{f}}}
\end{gathered}
$$

where $V$ is the volume of permeated water $(\mathrm{L}), A$ is the effective area of the membrane $\left(\mathrm{m}^{2}\right), \Delta t$ is the permeation time (h), $\Delta P$ is the applied pressure (bar), and $C_{\mathrm{f}}$ and $C_{\mathrm{p}}$ are the concentrations in the feed and permeate $\left(\mathrm{g} \mathrm{L}^{-1}\right)$, respectively. The salt concentration was measured with a conductivity meter (Thermo Scientific Orion Star A212). The solute concentration of direct/ reactive dyes was measured with a UV-Vis spectrophotometer (Shimadzu, Japan). All tests were run at $25 \pm 1{ }^{\circ} \mathrm{C}$.

2.3.6. Bacterial inhibition and viability. Membrane coupons of $4 \mathrm{~cm}^{2}$ were cut and sterilized by using ethanol. $4 \mathrm{~mL}$ suspension volumes of Escherichia coli (E. coli, $\sim 2 \times 10^{6} \mathrm{CFU}$ $\mathrm{mL}^{-1}$ ) were kept in contact with the membranes for $2 \mathrm{~h}$ at $30^{\circ} \mathrm{C}$ while shaking at $\sim 200 \mathrm{rpm}$. Diluted bacterial suspensions (100 $\mu \mathrm{L}$ ) obtained by the standard serial dilution method were spread onto agar plates, which were incubated overnight at 37 ${ }^{\circ} \mathrm{C}$. The number of colonies on the plates was determined by the plate colony-forming unit (CFU) count method, using plates with 3 to $1000 \mathrm{CFU}$.

\section{Results and discussion}

\subsection{Surface morphologies of membranes}

Photographed images of the pristine and modified membranes are displayed in Fig. S2. $\dagger$ A fast deposition functionalization is confirmed by the color changes on the white pristine PAN surface. PDA coatings exhibit a dark brown color, which is close to coatings prepared by the two-step approach. However, a dark green surface is obtained via the co-deposition of PDA and CuNPs, and the color becomes fainter with increasing codeposition times.

In order to further examine their surface morphologies, we conducted SEM images of the top layer for the pristine PAN and modified nanocomposite membranes (Fig. S3, $\dagger$ and 2). As seen from Fig. S3a and $b, \dagger$ the pristine PAN membrane presents a smooth and relatively loose surface morphology without macrovoids. After the deposition of CuNP suspension, CuNPs ranging from 20 to $50 \mathrm{~nm}$ are clearly present and successfully bound to the membrane surface (Fig. S3c and $d \dagger$ ). The shapes and sizes of these CuNPs are consistent with those found in the literature. ${ }^{54}$ The binding of CuNPs was achieved by the aid of the 

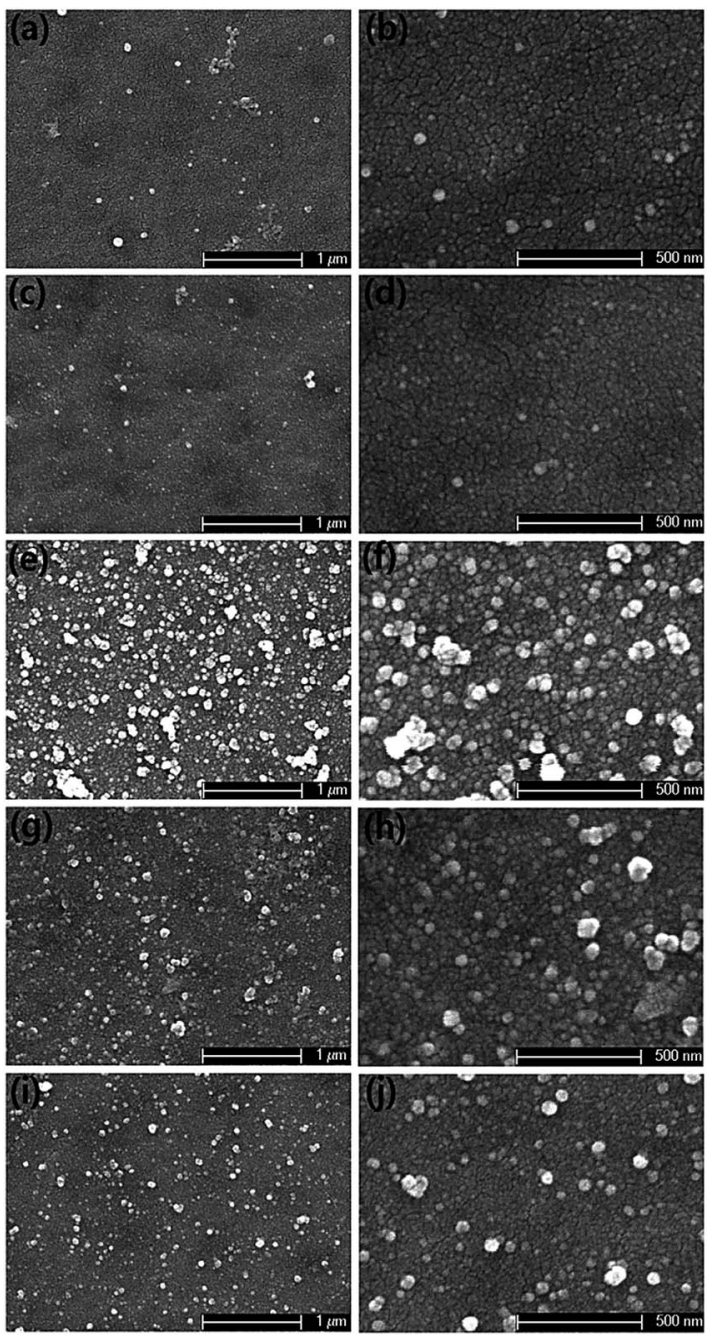

Fig. 2 Surface SEM images of the membranes modified with PDA and CuNPs in different magnifications: the modified membranes via twostep deposition: 30 min PDA deposition, followed by various CuNP deposition times of ( $a$ and b) $12 \mathrm{~h}$ and ( $c$ and d) $24 \mathrm{~h}$; the modified membranes with different co-deposition times of (e and f) $1.5 \mathrm{~h}$, ( $\mathrm{g}$ and h) $6 \mathrm{~h}$, and ( $\mathrm{i}$ and j) $12 \mathrm{~h}$.

high density of positive charges from PEI onto CuNP surfaces, thereby conferring stable electrostatic attraction to the negatively charged carboxyl groups on the support surface. ${ }^{55}$ Fig. S3e $\uparrow$ shows no apparent distinction in the surface modified with 30 min of PDA deposition compared to the pristine PAN membrane surface. However, the dense and compact surface morphology indicated in Fig. S3f $\dagger$ confirms a convinced PDA deposition onto PAN. Besides, the membrane surface exhibits a highly even morphology without any PDA aggregates or deposits, which was mainly attributed to the fast polymerization of dopamine and its uniform nucleation during the rapid deposition. Nevertheless, with $6 \mathrm{~h}$ of PDA loading, a small amount of nanoparticles appear on the membrane surface (Fig. S3g and $\mathrm{h} \dagger$ ). This presence is mainly ascribed to the aggregation of PDA, which implies that PDA deposition trigged by $\mathrm{H}_{2} \mathrm{O}_{2} / \mathrm{CuSO}_{4}$ should not be delayed for a long time.
Fig. 2 features the surface SEM images of membranes modified with PDA and CuNPs via the two different deposition methods. A small quantity of CuNPs was deposited on the HPAN surface after functionalization via the two-step deposition route (Fig. 2a-d). The lack of CuNPs indicates that two-step deposition is not only time-consuming, but also inefficient for surface loading. This inefficiency may be caused by an initially formed highly stable PDA layer that can hardly further selfpolymerize, thus markedly impairing interfacial adherence to CuNPs. Besides, some aggregates originally emerging on the PDA surface can lead to a sharp decrease in an even distribution of CuNPs. As presented in Fig. 2e-j, which features membranes functionalized by the co-deposition of PDA and CuNPs, abundant nanoparticles appeared on the coating layer with high uniformity and are tightly compact. Therefore, the co-deposition method evinces promises toward bridging the gaps from UF to NF. However, the amount of deposited nanoparticles presents a downward trend in its quantity as co-deposition time increases. This condition can be related to the compatibility and interfacial force among PEI-modified CuNPs, PDA, and the hydroxyl-functionalized surface. At the initial stage, as suggested by this inversely proportional trend, the positively charged PEI-modified CuNPs will primarily deposit onto the negatively charged HPAN surface before PDA can form a stable layer. PDA can also develop a strong linkage between CuNPs and the membrane surface to fill UF gaps. Still, PDA can adhere to CuNP surfaces, form a partial coverage, and impair its cationic surface charge. As the co-deposition time further progresses, CuNPs will inevitably be overlapped by PDA coatings, simultaneously making the membrane surface dense and compact.

EDX analysis was used to further investigate the elemental composition of the membrane surfaces modified by co-deposition. As seen from Fig. $\mathrm{S} 4, \dagger$ the measured $3.27 \mathrm{wt} \%$ of copper element verified the existence of CuNPs on the Co-NF-3 surface. Additionally, EDX mapping (Fig. S5†) clearly demonstrated the presence of copper on the membrane surfaces. In comparison to the two-step means, co-deposition of PDA and CuNPs provides a fast, effective technique for strongly binding CuNPs onto the porous polymeric support, aided by the rapid and homogeneous PDA deposition.

AFM images of the pristine and as-prepared membranes are displayed in Fig. 3. The pristine PAN membrane exhibits slightly rough peaks and valleys (Fig. 3a). After introduction of the PDA coater, the membrane surface becomes denser and more compact (Fig. 3b). The CuNP layer can also bridge the pristine PAN cavities, but, without the aid of PDA, some defective valleys can be still observed on the membrane surface (Fig. 3c). The surface roughness values of the membranes on $1 \times 1 \mu \mathrm{m}^{2}$ scans, as represented by mean root square roughness $\left(R_{\mathrm{rms}}\right)$, average roughness $\left(R_{\mathrm{a}}\right)$, and the maximum vertical difference between the highest and lowest points $\left(R_{\mathrm{m}}\right)$, are listed in Table 2.

As evinced by the roughness values, PDA $\left(R_{\mathrm{a}}=4.50 \mathrm{~nm} ; R_{\mathrm{rms}}\right.$ $=5.91 \mathrm{~nm})$ or CuNP $\left(R_{\mathrm{a}}=4.06 \mathrm{~nm} ; R_{\mathrm{rms}}=5.08\right)$ layers hardly change the PAN surface roughness or uniformity $\left(R_{\mathrm{a}}=4.38\right.$; $R_{\text {rms }}=5.52 \mathrm{~nm}$ ). This characteristic signifies that both modifications create smooth, homogeneous membrane surfaces. 
(a)
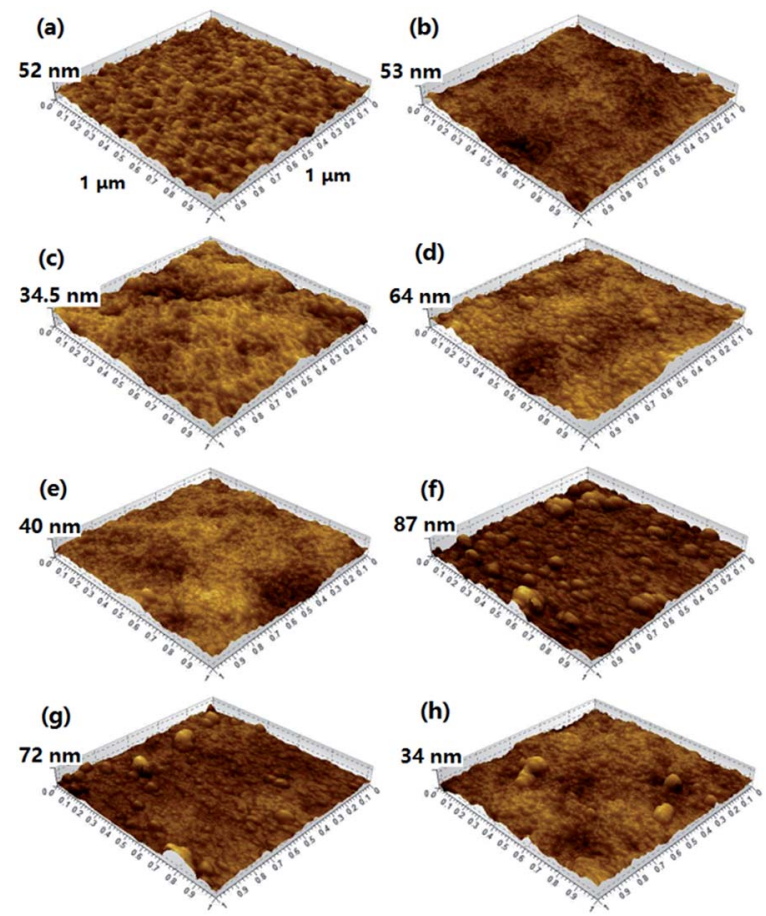

Fig. 3 AFM 3D images of pristine and modified membranes: (a) PAN, (b) PDA-HPAN-1, (c) CuNP-HPAN, the membranes modified with 30 min PDA followed by (d) $12 \mathrm{~h}$ and (e) $24 \mathrm{~h}$ deposition of CuNPs, the membranes modified with various co-deposition times: (f) $1.5 \mathrm{~h},(\mathrm{~g}) 6$ $h$, and (h) $24 \mathrm{~h}$.

Table 2 AFM surface roughness parameters of the pristine and modified membranes: $R_{\mathrm{a}}$ (average roughness), $R_{\mathrm{rms}}$ (root mean square roughness), and $R_{\mathrm{m}}$ (maximum vertical difference between the highest and lowest points)

\begin{tabular}{llcl}
\hline Membrane & $R_{\mathrm{a}}(\mathrm{nm})$ & $R_{\mathrm{rms}}(\mathrm{nm})$ & $R_{\mathrm{m}}(\mathrm{nm})$ \\
\hline PAN & 4.38 & 5.52 & 52.3 \\
PDA-HPAN-1 & 4.50 & 5.91 & 53.7 \\
CuNP-HPAN & 4.06 & 5.08 & 34.7 \\
NF-2 & 6.77 & 8.60 & 64.5 \\
NF-4 & 4.18 & 5.45 & 40.1 \\
Co-NF-1 & 8.77 & 12.0 & 87.7 \\
Co-NF-4 & 6.51 & 9.17 & 72.4 \\
Co-NF-5 & 3.77 & 4.84 & 34.3 \\
\end{tabular}

Such a small increase of the $R_{\mathrm{rms}}$ value in the two-step deposition membranes $\left(R_{\mathrm{rms}}=8.60 \mathrm{~nm}\right)$ demonstrates a low loading of CuNPs onto the PDA layer, which is also confirmed by SEM surface images. In comparison, Fig. $3 \mathrm{f}$ presents a rougher morphology $\left(R_{\mathrm{rms}}=12.0 \mathrm{~nm}\right)$ comprising plenty of nanoparticles on the membrane surface when functionalized by codeposition. Consistent with the SEM images, the superiority of co-deposition in firmly binding CuNPs is verified once again. The decreased surface roughness with increased deposition time is in accordance with the previously discussed SEM results. In conclusion, surface binding of PDA and CuNPs does not noticeably change the surface roughness of pristine membranes other than by CuNP deposition, holding a greater propensity to resist foulants.

\subsection{Surface hydrophilicity of membranes}

As reported by the previous literature, PDA and CuNP coatings have separately contributed to an enhancement of surface hydrophilicity. ${ }^{56,58}$ Otherwise, PDA/CuNP combined coatings have not been studied. Fig. 4A illustrates the dynamic water contact angles of PDA- and CuNP-modified HPAN membranes. The initial contact angle sharply decreases from $56.9^{\circ}$ to $47.6^{\circ}$ for hydrolyzed PAN modified by PDA for merely $30 \mathrm{~min}$. This result deviates slightly from a previous initial water contact angle of 30 min PDA deposition onto a polypropylene microporous membrane. The lower value $\left(c a .30^{\circ}\right)$ reported by the previous study was derived from the larger support membrane pore size used, which promoted the permeation of partial droplets. ${ }^{44}$ In addition, a distinct contact angle decline from $47.6^{\circ}$ to $32.2^{\circ}$ occurred in our study after two minutes in PDA-HPAN-1 samples. Similar dramatic downtrends also arose in other samples. The spreading of water droplets on the relatively compact membrane surfaces played a significant role in this contact angle decreasing trend more than water permeation did. Compared to the PDA layer, the CuNP coating granted HPAN even higher wettability, yielding an initial contact angle of $35.5^{\circ}$. This property is largely based on the favorable combination of the imino groups of PEI with the hydrophilic CuNPs.

Fig. 4B displays the dynamic water contact angles of PDA/ CuNP-modified HPAN membranes via two-step deposition. After CuNP loading onto the PDA-modified membranes, each sample clearly manifested sharper decline trends over time compared to the membranes separately modified with PDA or CuNPs only. The initial contact angle in two-step modified membranes further reduced from $35.3^{\circ}$ to $30.0^{\circ}$ as CuNP
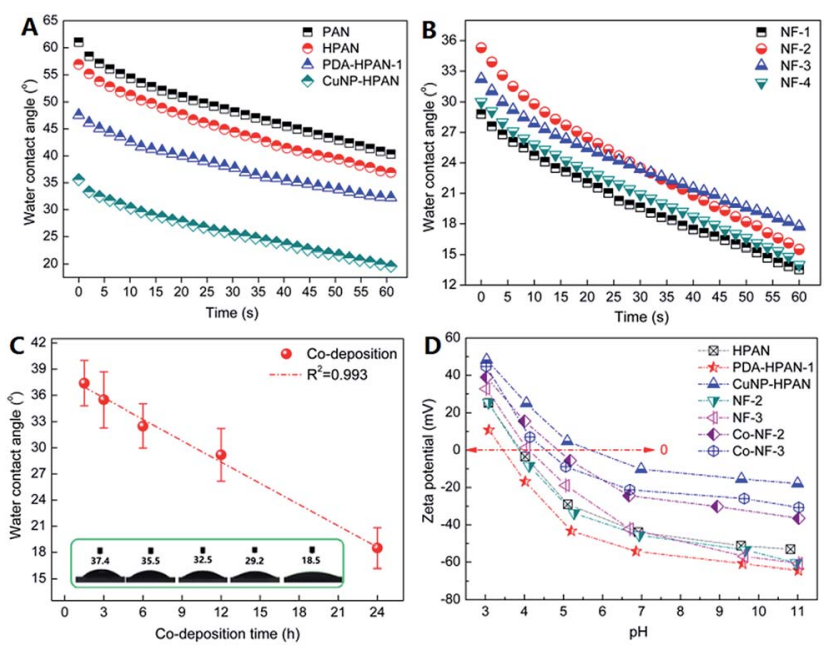

Fig. 4 (A) Dynamic water contact angles of pristine and PDA- and CuNP-modified HPAN membranes, (B) dynamic water contact angles of PDA-CuNP-HPAN membranes via two-step deposition, (C) static water contact angles of PDA-CUNP-HPAN membranes prepared by co-deposition as a function of immersion time, and (D) zeta potential of the modified membranes versus $\mathrm{pH}$ value. 
coating time increased from 12 to $24 \mathrm{~h}$. These results reveal that stronger wettability can be acquired when membranes are modified with both PDA and CuNPs. The static water contact angles of membranes modified with various co-deposition times are highlighted in Fig. 4C. Remarkably, the water contact angles linearly decreased with co-deposition time. The plausible explanation to this phenomenon is accredited to the smoother morphologies that originated with increasing co-deposition times, thus inducing the stronger hydrophilicity of the membrane surface. In summary, we conclude that the codeposition procedure can confer advantageous hydrophilicity to pristine membranes, outperforming the traditional two-step deposition methods.

\subsection{Charged characteristics of membrane surfaces}

With regard to NF separation mechanisms, low molecular weight (200-1000 Da) uncharged solutes are primarily retained by the size exclusion effect. On the other hand, the Donnan effect plays an important role in the diverse selectivity of charged solutes, especially for multivalent salts. Therefore, charged membrane surfaces will facilitate the passing of oppositely charged solutes and screen the passage of similarly charged solutes through the membrane.

In this work, zeta potential measurements were performed to evaluate the surface charge of the as-prepared membranes (Fig. 4D). HPAN shows an extensive negative zeta potential over a wide $\mathrm{pH}$ range (3.9-11), which is predominantly due to the carboxyl groups generated by the basic hydrolysis of PAN nitrile groups. Surface charge substantially changed with different coatings of PDA and CuNPs. For instance, zeta potential values that are more strongly negative were obtained when HPAN was surface functionalized by the PDA layer, and the isoelectric point shifted from 3.9 to 3.4. Conversely, after the binding of CuNPs onto HPAN, the isoelectric point increased to 5.7, drastically enhancing zeta potential values to be more neutral to assist multivalent salt separation. As previously mentioned, multiple cationic imine groups arising from PEI exist on the surface of the CuNPs, causing the nanoparticles to carry a highly positive charge density.

The design of introducing CuNPs to a PDA layer was developed to achieve a neutral surface, which can facilitate high salt permeation through such membranes. For two-step deposition membranes, the increased density of surface negative charge from the PDA layer was partially compensated by the positively charged PEI-modified CuNPs. These membranes had an isoelectric point at 4.1, slightly higher than an isoelectric point of HPAN at 3.9. Surface charge neutralization was even more splendid when the co-deposition strategy was applied, leading to an isoelectric point at 4.8. The formation of the tightly packed structure and increasing attached CuNP concentration enable a more neutral charge of the membrane surface, giving rise to enhanced salt permeation.

\subsection{Water permeability of membranes}

Fig. 5 manifests the average water permeability of each pristine and modified PAN membrane among four various pressures (1-8 bar). The pristine PAN membrane revealed a high water permeability of $208.4 \mathrm{~L} \mathrm{~m}^{-2} \mathrm{~h}^{-1}$ bar $^{-1}$. After hydrolysis with $\mathrm{NaOH}$, the corresponding value slightly improved to $230.7 \mathrm{~L} \mathrm{~m}^{-2} \mathrm{~h}^{-1} \mathrm{bar}^{-1}$ as a result of the increased surface hydrophilicity. After $30 \mathrm{~min}$ of PDA deposition, the water permeability notably reduced to $15.8 \mathrm{~L} \mathrm{~m}^{-2} \mathrm{~h}^{-1} \mathrm{bar}^{-1}$. This result confirms that a strong and effective PDA layer covered the UF support via $\mathrm{CuSO}_{4} / \mathrm{H}_{2} \mathrm{O}_{2}$-triggered fast PDA deposition. After extending the PDA deposition time to $6 \mathrm{~h}$ to explore the effect of PDA deposition time on water permeability, the value $\left(8.1 \mathrm{~L} \mathrm{~m}^{-2} \mathrm{~h}^{-1} \mathrm{bar}^{-1}\right)$ nearly diminished by half compared with that of membranes with 30 min PDA coating. This decrease suggests that PDA functionalization is a time-dependent process with increased deposition times leading to denser membranes. Interestingly, HPAN membranes coated with CuNPs without PDA achieved the lowest permeability: $5.6 \mathrm{~L} \mathrm{~m}^{-2} \mathrm{~h}^{-1} \mathrm{bar}^{-1}$. This deficiency is likely ascribed to the strong binding of CuNPs via electrostatic attraction into the membrane surface pores.

DI water permeability attained using the two-step deposition and co-deposition membranes is shown in Fig. 5B. As seen from this figure, for two-step coating, the water permeability was improved from 16.7 to $18.6 \mathrm{~L} \mathrm{~m}^{-2} \mathrm{~h}^{-1}$ bar $^{-1}$ with increasing CuNP deposition time from 12 to $24 \mathrm{~h}$. As confirmed by the SEM images (Fig. S3e and $f^{\dagger}$ ), the PDA coating creates a smooth and slightly dense surface. A continuously growing CuNP binding onto the PDA layer with increasing coating time enables a more hydrophilic surface and an increase of water permeability. For membranes modified by co-deposition of PDA and CuNPs, the water permeability eminently reached the highest value $(25.5 \mathrm{~L}$ $\mathrm{m}^{-2} \mathrm{~h}^{-1}$ bar $^{-1}$ ) with $1.5 \mathrm{~h}$ co-deposition. The presence of CuNPs physically impedes the formation of a dense coating layer originating from dopamine self-polymerization, mainly owing to the relatively poor compatibility between inorganic nanoparticles and organic PDA. Such a formation behavior results in a loose and hydrophilic surface, thereby facilitating a high water flux. However, water permeability also followed a declining trend with lengthened co-deposition times. This trend mostly likely ensued from the further formation of a more compact and thicker top layer, increasing hydraulic resistance through the membrane to lower the water permeability to $11.1 \mathrm{~L} \mathrm{~m}^{-2} \mathrm{~h}^{-1}$ bar $^{-1}$ with 24 h co-deposition. Such results indicate that the co-

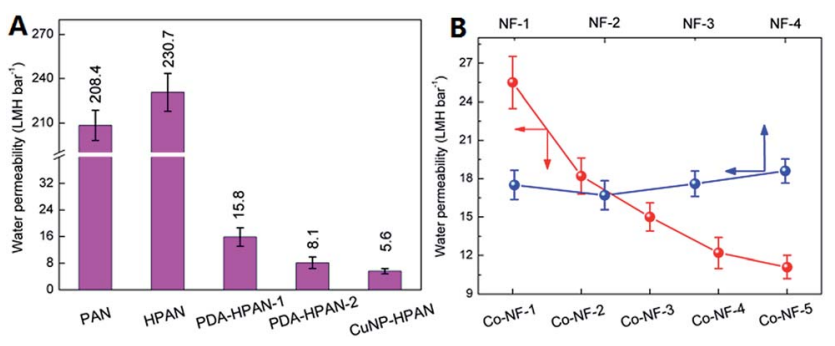

Fig. 5 DI water permeability of (A) membranes used for comparison and (B) two-step deposition and co-deposition membranes. Reported values represent the average water permeability over four varied pressures (PAN and HPAN: 1-4 bar; other membranes: 2-8 bar). LMH bar $^{-1}$ is short for $\mathrm{L} \mathrm{m}^{-2} \mathrm{~h}^{-1}$ bar $^{-1}$. 
deposition method, particularly with faster treating time, leads to the most advantageous water permeability.

\subsection{Separation properties of membranes}

3.5.1. Dye retention. Three types of dye solution, direct red 23 (DR23), Congo red (CR), and reactive blue 2 (RB2), were used to explore the NF separation performance of the membranes. Fig. 6 shows the effect of different coating layers and deposition times on the retention and permeation flux of dye solutions. Surprisingly, the pristine PAN membrane (MWCO $=75 \mathrm{kDa}$ ) attained a fairly high retention for CR and DR23 direct dyes with MW ranging from $696.7(R=97.5 \%)$ to 813.7 Da $(R=98.3 \%)$, respectively (Fig. 6A). Previous studies demonstrated that dye molecules were inclined to form aggregates and clusters due to hydrophobic interactions included in aggregation processes. ${ }^{\mathbf{9}}$ Hamada et al. also reported that the hydrating water molecules surrounding the dyes contributed to this process. ${ }^{60}$ Moreover, dye molecules often exhibit very low diffusivity. For instance, the diffusivity of $\mathrm{CR}$ at $30{ }^{\circ} \mathrm{C}$ equalled that of poly(ethylene glycol) (MW > $2000 \mathrm{Da}) .{ }^{61}$ All of these studies reveal that dye aggregation is quite common in aqueous solution, allowing PAN to reject much of the direct dyes. The high retention of direct dyes greatly depends on the increased molecular size of dye clusters in aqueous solution. In addition to size exclusion, the Donnan effect also plays a significant role in the high rejection of negatively charged dyes. However, the pristine PAN membrane had quite low reactive dye retention (RB2, 52.9\%).

After individual coating with either PDA or CuNPs, an obvious increase in dye retention was observed (Fig. 6A). Increasing the PDA deposition time from $30 \mathrm{~min}$ to $6 \mathrm{~h}$ improved the retention of DR23 (99.4\% to $99.7 \%)$, CR (99.2\% to $99.5 \%$ ), and RB2 (81.9\% to $90.3 \%)$. These results demonstrate that PDA coatings substantially boost separation properties of composite membranes. Nonetheless, the permeation flux
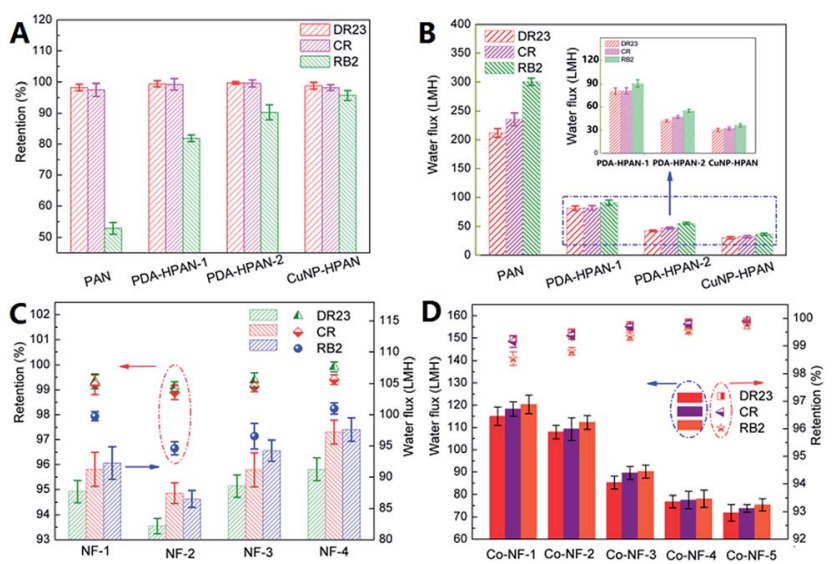

Fig. 6 Dye retention and permeation flux of ( $A$ and $B$ ) the pristine membranes and membranes modified with PDA or CuNP coatings, (C) the modified membranes via two-step deposition, and (D) the modified membranes via co-deposition. Temperature was maintained throughout the filtrations at $25 \pm 1^{\circ} \mathrm{C}$. The influent dye concentration used was $0.5 \mathrm{~g} \mathrm{~L}^{-1}$. Pressures applied were 2 bar for the PAN and HPAN membranes and 8 bar for the other membranes. distinctly reduced nearly by half for the dye solutions as deposition time increased from $30 \mathrm{~min}$ to $6 \mathrm{~h}$ (Fig. 6B). Thus, we chose $30 \mathrm{~min}$ for PDA functionalization in the two-step deposition method. CuNP-functionalized HPAN (CuNP-HPAN) without PDA achieved a relatively low flux and rejection. This outcome is mainly due to the less negatively charged surface and partially defect voids. Additionally, such a low permeation flux hinders the practicality of dye desalination.

Fig. 6C presents the dye retention and permeation flux of the membranes modified by two-step deposition. For membranes with $30 \mathrm{~min}$ of PDA coating, dye retention manifested an upward trend as CuNP coating increased from 12 to $24 \mathrm{~h}$. With the increased coating of positively charged CuNPs, a more neutral surface charge formed along with a denser and more compact surface structure to facilitate dye rejection. RB2 retention even enhanced to $98.3 \%$ when CuNP coating took place for $24 \mathrm{~h}$. Despite the functionality of high dye retention, the two-step deposition process and $24 \mathrm{~h}$ CuNP coating is ultimately still time-consuming.

The dye separation performance of the membranes modified via co-deposition is showcased in Fig. 6D. As mentioned, the top layer becomes denser, more compact, and thicker with increasing co-deposition time. This formation mechanism is similar to two-step coating, as PDA and CuNPs are continuously deposited onto the membrane surface to boost membrane resistance. Higher dye rejection is generally promoted based on size exclusion by the denser surfaces. This enhancement, however, comes at the cost of decreased water flux. While the different membranes consistently accomplished ultra-high retention of direct dyes, the rejection and permeation flux for the reactive dye RB2 should especially be emphasized. Its retention increased from $98.5 \%$ to $99.8 \%$ when increasing the co-deposition time from 1.5 to $24 \mathrm{~h}$, whereas the corresponding flux decreased from 120.3 to $75.4 \mathrm{~L} \mathrm{~m}^{-2} \mathrm{~h}^{-1}$ under 8 bar. A compromise among time, selectivity, and flux should be further considered for potential applications. Overall, high dye retention and permeation flux render NF co-deposition composite membranes competitive for dye wastewater treatment.

3.5.2. Salt permeation. Resource reuse and recovery (e.g., dye purification and salt reuse) has been an integral aspect of textile wastewater treatment. Loose NF membranes must therefore be tailored to effectively purify dyes by gaining high salt permeability. Fig. 7 summarizes the salt retention $\left(\mathrm{MgCl}_{2}\right.$, $\mathrm{NaCl}$, and $\mathrm{Na}_{2} \mathrm{SO}_{4}$ ) and permeation flux of the modified membranes. The PDA coating conferred a fairly high $\mathrm{Na}_{2} \mathrm{SO}_{4}$ retention to the hydrolyzed PAN membrane; the retention improved from $68.8 \%$ to $79.8 \%$ as coating time increased from $30 \mathrm{~min}$ to $6 \mathrm{~h}$. The order of decreasing salt retention $\left(\mathrm{Na}_{2} \mathrm{SO}_{4}>\right.$ $\mathrm{NaCl}>\mathrm{MgCl}_{2}$ ) identifies a negatively charged membrane, which corroborates the zeta potential results. Additionally, $\mathrm{MgCl}_{2}$ retention slightly increased for $6 \mathrm{~h}$ PDA deposition instead of 30 min, emphasizing the formation of a dense and compact surface which enables higher salt rejection. An enhanced salt retention could essentially be explained by the combination of steric and electrostatically repulsive effects. HPAN membranes functionalized only by CuNPs performed a very low salt retention for all salts. The defect voids existing on the membrane 

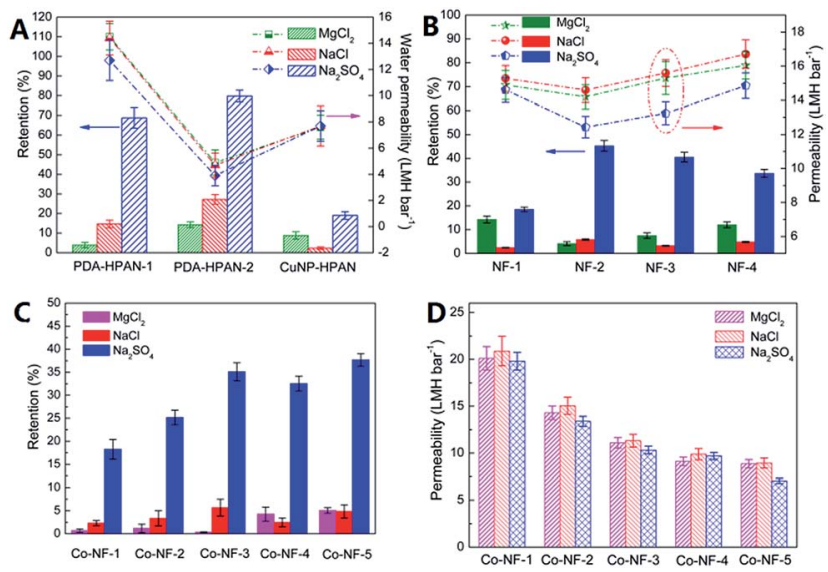

Fig. 7 Salt retention and permeation flux of the modified membranes: (A) PDA- and CuNP-modified membranes, (B) membranes prepared via two-step deposition, and ( $C$ and $D$ ) membranes prepared via codeposition. The influent salt concentration used was $1.0 \mathrm{~g} \mathrm{~L}^{-1}$.

surface most likely caused the membrane to be incompatible for salt rejection. The highest permeation flux of the membranes used for comparison was observed for PDA-HPAN1, making 30 min PDA coating an optimal design parameter for two-step modification.

As shown in Fig. 7B, NF-1 has a low retention of $\mathrm{Na}_{2} \mathrm{SO}_{4}$ $(18.5 \%)$ and $\mathrm{NaCl}(2.5 \%)$. As previously mentioned in Table $\mathrm{S} 1, \uparrow$ NF-1 was prepared by 20 min PDA coating followed by $24 \mathrm{~h}$ CuNP deposition. Such a short PDA coating time instigates difficulties in fully covering gaps or attaching CuNPs without defect voids on the membrane surface. On the basis of $30 \mathrm{~min}$ of PDA coating, it was apparent that the $\mathrm{Na}_{2} \mathrm{SO}_{4}$ retention remarkably declined after introducing the CuNP layer. As confirmed by zeta potential measurements, after CuNP coating, the membrane surface became less negatively charged due to the multitude of positive charges on the CuNP surface. The decreased salt retention by this loose NF membrane primarily stemmed from less electrostatic repulsion existing between the bivalent co-ion $\left(\mathrm{SO}_{4}{ }^{2-}\right)$ and the membrane surface. From NF-2 to NF-4, the rejection of $\mathrm{Na}_{2} \mathrm{SO}_{4}$ slightly reduced with the increase of deposited positively charged CuNPs that diminish the Donnan effect. The enhanced water permeability from NF-2 to NF-4 is mainly attributed to the improved hydrophilicity of the modified surface.

Fig. 7C and D present the salt retention and water flux of the membranes modified via co-deposition. The $1.5 \mathrm{~h}$ co-deposition membrane (Co-NF-1) achieved the lowest overall salt retention $\left(0.7 \% \mathrm{MgCl}_{2}, 2.3 \% \mathrm{NaCl}\right.$, and $\left.18.3 \% \mathrm{Na}_{2} \mathrm{SO}_{4}\right)$ and highest permeability $\left(\sim 20 \mathrm{~L} \mathrm{~m}^{-2} \mathrm{~h}^{-1}\right.$ bar $\left.^{-1}\right)$ among any of the modified membranes. This high salt permeation was primarily due to the combination of the loose surface structure, faint negative charge, and different hydrated ionic radii. The loose surface induced by the inorganic CuNPs significantly increased salt permeation, and the increased surface area stemming from this morphology may also accelerate water permeability. However, both salt permeation and water flux visibly reduced with increased co-deposition times. For instance, the permeability exhibited a sharp decline from 19.8 to $7.1 \mathrm{~L} \mathrm{~m}^{-2} \mathrm{~h}^{-1} \mathrm{bar}^{-1}$ for $\mathrm{Na}_{2} \mathrm{SO}_{4}$ solution with co-deposition time increasing from 1.5 to $24 \mathrm{~h}$. This observation is probably because of the enhanced membrane resistance induced by the formation of denser and more compact top layer structures. From the perspective of both selectivity and permeability, the results confirm that co-deposition of PDA and CuNPs is superior to the two-step approach. Besides, co-deposition is a facile, time-saving, and cost-effective strategy for the design of loose NF membranes.

Fig. 8 reveals the mechanism of salt permeation through the modified membranes. The salt and water transport properties through the composite membranes are also influenced by the morphology, size, and polymer interface compatibility and adhesion of the functionalized NPs. The relatively dense structure and strong negative charge of the PDA coating screens extensive amounts of sulfate ions, greatly increasing $\mathrm{Na}_{2} \mathrm{SO}_{4}$ retention. The favorable hydrophilicity of PDA still encourages water transport as well. In comparison, after the CuNP suspension is mixed with PDA solution during co-deposition, the relatively poor compatibility between them results in some interface voids in the vicinity of CuNPs. These voids form a relatively loose PDA-based matrix structure. CuNPs covered with multiple positively charged PEI molecules can also largely neutralize the negative charge density of PDA, thus promoting hydrated ion transport. Hence, the sulfate ions tend to pass through the positively charged membrane channel as depicted in Fig. 8B. Likewise, the higher hydrophilicity of the co-deposition membranes compared to the PDA coating alone promotes water permeability further.

3.5.3. Comparison to other established membranes. In order to further compare the overall performance of the NF membranes in the water permeability and selectivity of dyes and salts, Table $\mathrm{S} 2 \dagger$ summarizes a comparison of established commercial and synthesized membranes to these NF membranes prepared by co-deposition in this study.

In other studies, a thin-film compact membrane with a polyamide layer generally exhibited relatively low water permeability (7.0-8.5 $\mathrm{LMH} \mathrm{bar}^{-1}$ ). Though surface dip-coating
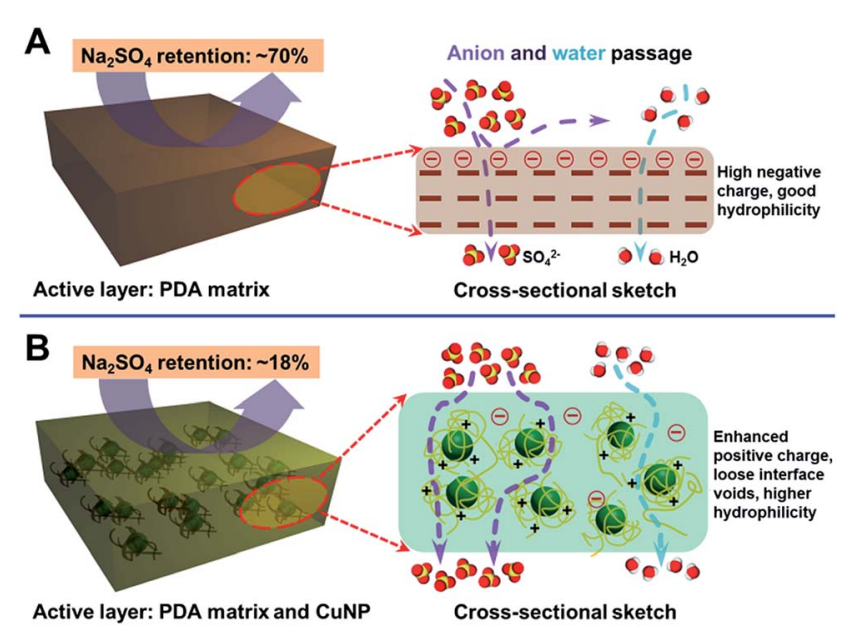

Fig. 8 The mechanisms of salt permeation through (A) membranes modified by PDA only and (B) membranes modified via co-deposition. 
and crosslinking strategies improved the water permeability to 10.8 $\mathrm{LMH}$ bar $^{-1}$, these membranes showed a rather high retention of $\mathrm{Na}_{2} \mathrm{SO}_{4}$ (85.5-98.5\%) and $\mathrm{NaCl}$ (28.8-64.4\%). Blending nanosheets with polymers to construct loose NF membranes is a facile and cost-effective method that can greatly reduce bivalent salt retention $\left(\sim 10.0 \%\right.$ for $\left.\mathrm{Na}_{2} \mathrm{SO}_{4}\right)$. However, two existing drawbacks still remain as enormous challenges in these methods: low dye retention and water permeability. Commercially available membranes (Sepro NF 2A and NF 6 from Ultura, USA) displayed effective performances in water permeability and selectivity of direct dyes and salts. High dye retention and $\mathrm{NaCl}$ permeation make these loose membranes suitable for the separation of dye/ $\mathrm{NaCl}$ mixtures, but these $\mathrm{NF}$ membranes typically face difficulties in achieving high $\mathrm{Na}_{2} \mathrm{SO}_{4}$ permeation.

In this study, $3 \mathrm{~h}$ co-deposition of PDA and CuNPs could be highlighted due to producing membranes with the highest water permeability (18.2 $\mathrm{LMH} \mathrm{bar}^{-1}$ ) and compared with similar state-of-the-art membranes. Furthermore, the achieved ultra-high dye retention and low $\mathrm{Na}_{2} \mathrm{SO}_{4}$ rejection validated their competitive potential in the application of dye/salt fractionation.

\subsection{Antimicrobial activity}

Fig. 9 describes the strong antimicrobial activities of the modified membranes. To quantitatively analyze the bactericidal ability, the viable cell counting technique was applied in this study. The PDA-HPAN-1 membrane prepared by the fast PDA
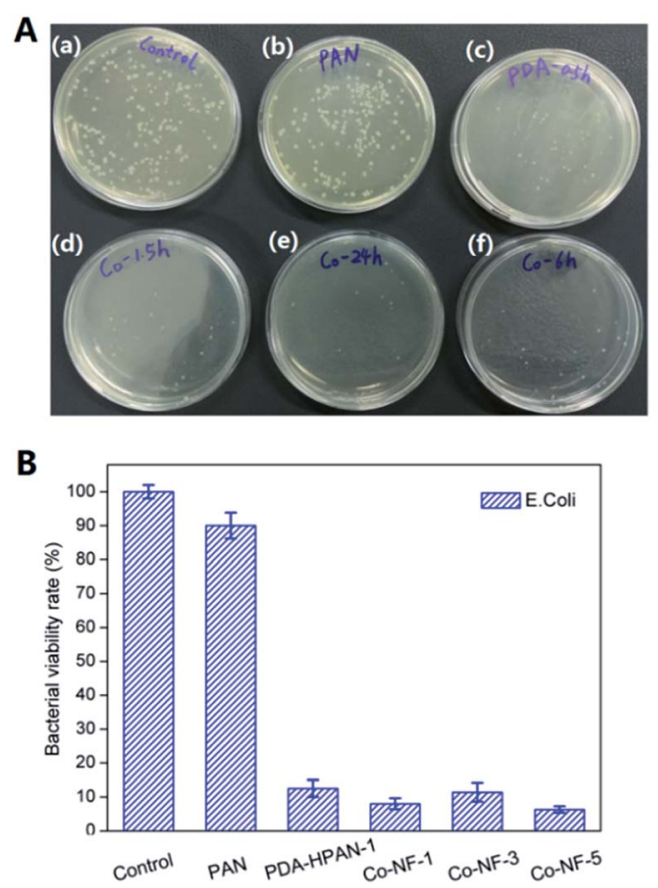

Fig. 9 (A) Demonstrated antibacterial properties of the membranes by the plate counting method: (a) control, (b) PAN, (c) PDA-HPAN-1, (d) Co-NF-1, (e) Co-NF-5, and (f) Co-NF-3; (B) quantified antimicrobial ability of the nascent PAN, PDA-modified, and co-deposition-modified membranes. deposition exhibited a high biocidal ability against $E$. coli. This trait may be attributed to the release of copper ions from the PDA layer. Relative to the control sample, the modified membranes reduced $92.0 \%, 88.6 \%$, and $93.7 \%$ of $E$. coli when $1.5 \mathrm{~h}, 6 \mathrm{~h}$, and $24 \mathrm{~h}$ of the co-deposition modification method were applied, respectively. Such distinct reductions in E. coli colonies demonstrate the excellent antimicrobial properties of the modified membranes.

Although CuNPs show considerable toxicity to bacteria, viruses, and fungi, it still remains challenging to elucidate accurate antibacterial mechanisms. ${ }^{62}$ The biocidal activity is, however, based on the mode of contact-killing. As reviewed by Hajipour et al., several factors that influence the toxicity of CuNPs include the concentration of nanoparticles and bacteria, temperature, aeration, and $\mathrm{pH}^{62}$ These factors can largely affect CuNP agglomeration, which could decrease the available contact area with bacteria. Furthermore, some studies emphasize the formation of reactive oxygen species (ROS) produced by CuNPs and copper ions, which severely damage the cell, DNA, and mitochondria of bacteria. ${ }^{63}$ The depletion of copper ions is also considered to play an important role in bacteria damage. ${ }^{64}$

\section{Conclusions}

Loose NF membranes prepared by the fast deposition of PDA and CuNPs yielded remarkable water permeability, dye retention, salt permeation, and antimicrobial activity. Favorable surface properties, such as high hydrophilicity and low roughness, were also verified by a series of characterizations. Two preparation techniques were proposed: two-step deposition and co-deposition of PDA and CuNPs. In both cases, PDA deposited rapidly and uniformly onto a UF support, bridging surface cavities into a loose NF membrane and subsequently binding PEI-modified CuNPs firmly. The most optimal modification parameters for the fractionation of dye/salt mixtures were identified as $3 \mathrm{~h}$ of PDA and CuNP co-deposition. This quick codeposition time used reduces typical times required by previous membrane applications using either PDA or CuNPs. Moreover, the relatively inexpensive cost of copper as an antimicrobial agent allows copper nanoparticles to be used as a practical antibiofouling nanomaterial. Remarkably, the ultra-high dye retention and salt permeability achieved establishes the membranes in this study as competitive and practical solutions for textile wastewater treatment among other potential NF applications. More importantly, this rapid, facile co-deposition strategy offers possibilities in the strong binding of other functional nanoparticles (i.e., $\mathrm{Ag}, \mathrm{ZnO}$, and graphene oxide) with enhanced antimicrobial properties onto the membrane surface.

\section{Author contribution}

Junyong Zhu, Jiuyang Lin and Bart Van der Bruggen conceived the research. The primary experimental work was conducted by Junyong Zhu and Adam Uliana. Jing Wang, Shushan Yuan, Jian Li, Miaomiao Tian, and Alexander Volodin performed sample analyses and characterizations. Kenneth Simoens led the 
antibacterial tests. All authors contributed to manuscript writing and editing.

\section{Acknowledgements}

J. Y. Zhu would like to acknowledge the support provided by China Scholarship Council (CSC) of the Ministry of Education, P. R. China. Ultura, USA, is greatly acknowledged for providing the ultrafiltration membrane samples.

\section{References}

1 J. Lin, W. Ye, H. Zeng, H. Yang, J. Shen, S. Darvishmanesh, P. Luis, A. Sotto and B. Van der Bruggen, J. Membr. Sci., 2015, 477, 183-193.

2 J. Zhu, M. Tian, J. Hou, J. Wang, J. Lin, Y. Zhang, J. Liu and B. Van der Bruggen, J. Mater. Chem. A, 2016, 4, 1980-1990.

3 L. Wang, S. Ji, N. Wang, R. Zhang, G. Zhang and J.-R. Li, J. Membr. Sci., 2014, 452, 143-151.

4 L. Yu, Y. Zhang, Y. Wang, H. Zhang and J. Liu, J. Hazard. Mater., 2015, 287, 373-383.

5 A. W. Mohammad, Y. H. Teow, W. L. Ang, Y. T. Chung, D. L. Oatley-Radcliffe and N. Hilal, Desalination, 2015, 356, 226-254.

6 B. Van der Bruggen, B. Daems, D. Wilms and C. Vandecasteele, Sep. Purif. Technol., 2001, 22-23, 519-528.

7 J. Lin, C. Y. Tang, C. Huang, Y. P. Tang, W. Ye, J. Li, J. Shen, R. Van den Broeck, J. Van Impe, A. Volodin, C. Van Haesendonck, A. Sotto, P. Luis and B. Van der Bruggen, J. Membr. Sci., 2016, 501, 1-14.

8 J. Zhu, M. Tian, Y. Zhang, H. Zhang and J. Liu, Chem. Eng. J., 2015, 265, 184-193.

9 L. Yu, J. Deng, H. Wang, J. Liu and Y. Zhang, ACS Sustainable Chem. Eng., 2016, 4, 3292-3304.

10 J. Lin, C. Y. Tang, W. Ye, S.-P. Sun, S. H. Hamdan, A. Volodin, C. V. Haesendonck, A. Sotto, P. Luis and B. Van der Bruggen, J. Membr. Sci., 2015, 493, 690-702.

11 J. Zhu, Y. Zhang, M. Tian and J. Liu, ACS Sustainable Chem. Eng., 2015, 3, 690-701.

12 L. Xing, N. Guo, Y. Zhang, H. Zhang and J. Liu, Sep. Purif. Technol., 2015, 146, 50-59.

13 J. Wang, Y. Zhang, J. Zhu, J. Hou, J. Liu and B. Van der Bruggen, J. Membr. Sci., 2016, 510, 27-37.

14 J. Zhu, N. Guo, Y. Zhang, L. Yu and J. Liu, J. Membr. Sci., 2014, 465, 91-99.

15 T.-S. Chung, L. Y. Jiang, Y. Li and S. Kulprathipanja, Prog. Polym. Sci., 2007, 32, 483-507.

16 F. Perreault, M. E. Tousley and M. Elimelech, Environ. Sci. Technol. Lett., 2014, 1, 71-76.

17 Y. Li, Y. Su, J. Li, X. Zhao, R. Zhang, X. Fan, J. Zhu, Y. Ma, Y. Liu and Z. Jiang, J. Membr. Sci., 2015, 476, 10-19.

18 Y.-F. Mi, Q. Zhao, Y.-L. Ji, Q.-F. An and C.-J. Gao, J. Membr. Sci., 2015, 490, 311-320.

19 W.-P. Zhu, J. Gao, S.-P. Sun, S. Zhang and T.-S. Chung, J. Membr. Sci., 2015, 487, 117-126.

20 H. Dong, L. Wu, L. Zhang, H. Chen and C. Gao, J. Membr. Sci., 2015, 494, 92-103.
21 T. He, M. Frank, M. H. V. Mulder and M. Wessling, J. Membr. Sci., 2008, 307, 62-72.

22 F. Liu, B.-r. Ma, D. Zhou, L.-J. Zhu, Y.-Y. Fu and L.-x. Xue, React. Funct. Polym., 2015, 86, 191-198.

23 W. Ding, J. Cai, Z. Yu, Q. Wang, Z. Xu, Z. Wang and C. Gao, J. Mater. Chem. A, 2015, 3, 20118-20126.

24 Q. Chen, P. Yu, W. Huang, S. Yu, M. Liu and C. Gao, J. Membr. Sci., 2015, 492, 312-321.

25 H. Lee, S. M. Dellatore, W. M. Miller and P. B. Messersmith, Science, 2007, 318, 426-430.

26 S. M. Kang, N. S. Hwang, J. Yeom, S. Y. Park, P. B. Messersmith, I. S. Choi, R. Langer, D. G. Anderson and H. Lee, Adv. Funct. Mater., 2012, 22, 2949-2955.

27 Y. Liu, K. Ai and L. Lu, Chem. Rev., 2014, 114, 5057-5115.

28 H.-C. Yang, K.-J. Liao, H. Huang, Q.-Y. Wu, L.-S. Wan and Z.-K. Xu, J. Mater. Chem. A, 2014, 2, 10225-10230.

29 D. J. Miller, P. A. Araújo, P. B. Correia, M. M. Ramsey, J. C. Kruithof, M. C. M. van Loosdrecht, B. D. Freeman, D. R. Paul, M. Whiteley and J. S. Vrouwenvelder, Water Res., 2012, 46, 3737-3753.

30 Y. Li, Y. Su, X. Zhao, X. He, R. Zhang, J. Zhao, X. Fan and Z. Jiang, ACS Appl. Mater. Interfaces, 2014, 6, 5548-5557.

31 Y. Liao, R. Wang and A. G. Fane, J. Membr. Sci., 2013, 440, 77-87. 32 R.-X. Zhang, L. Braeken, P. Luis, X.-L. Wang and B. Van der Bruggen, J. Membr. Sci., 2013, 437, 179-188.

33 L. Shao, Z. X. Wang, Y. L. Zhang, Z. X. Jiang and Y. Y. Liu, J. Membr. Sci., 2014, 461, 10-21.

34 H. Shi, Y. He, Y. Pan, H. Di, G. Zeng, L. Zhang and C. Zhang, J. Membr. Sci., 2016, 506, 60-70.

35 H.-C. Yang, J.-K. Pi, K.-J. Liao, H. Huang, Q.-Y. Wu, X.-J. Huang and Z.-K. Xu, ACS Appl. Mater. Interfaces, 2014, 6, 12566-12572.

36 Y. Lv, H.-C. Yang, H.-Q. Liang, L.-S. Wan and Z.-K. Xu, J. Membr. Sci., 2016, 500, 265-271.

37 Z.-X. Wang, C.-H. Lau, N.-Q. Zhang, Y.-P. Bai and L. Shao, J. Mater. Chem. A, 2015, 3, 2650-2657.

38 C. Cheng, S. Li, W. Zhao, Q. Wei, S. Nie, S. Sun and C. Zhao, J. Membr. Sci., 2012, 417-418, 228-236.

39 J. Zhao, Y. Su, X. He, X. Zhao, Y. Li, R. Zhang and Z. Jiang, J. Membr. Sci., 2014, 465, 41-48.

40 M. Li, J. Xu, C.-Y. Chang, C. Feng, L. Zhang, Y. Tang and C. Gao, J. Membr. Sci., 2014, 459, 62-71.

41 S. Kasemset, A. Lee, D. J. Miller, B. D. Freeman and M. M. Sharma, J. Membr. Sci., 2013, 425-426, 208-216.

42 G. Han, S. Zhang, X. Li, N. Widjojo and T.-S. Chung, Chem. Eng. Sci., 2012, 80, 219-231.

43 H. W. Kim, B. D. McCloskey, T. H. Choi, C. Lee, M.-J. Kim, B. D. Freeman and H. B. Park, ACS Appl. Mater. Interfaces, 2013, 5, 233-238.

44 C. Zhang, Y. Ou, W.-X. Lei, L.-S. Wan, J. Ji and Z.-K. Xu, Angew. Chem., Int. Ed., 2016, 128, 3106-3109.

45 J. Wingender, T. R. Neu and H.-C. Flemming, in Microbial extracellular polymeric substances, Springer, Berlin, Germany, 1999, ch. 1, pp. 1-19.

46 M. S. Rahaman, H. Therien-Aubin, M. Ben-Sasson, C. K. Ober, M. Nielsen and M. Elimelech, J. Mater. Chem. $B, 2014,2,1724-1732$. 
47 J. Jiang, L. Zhu, L. Zhu, H. Zhang, B. Zhu and Y. Xu, ACS Appl. Mater. Interfaces, 2013, 5, 12895-12904.

48 Y. Sui, X. Gao, Z. Wang and C. Gao, J. Membr. Sci., 2012, 394395, 107-119.

49 S. Chernousova and M. Epple, Angew. Chem., Int. Ed., 2013, 52, 1636-1653.

50 L. Yu, Y. Zhang, B. Zhang and J. Liu, Sci. Rep., 2014, 4, 4551.

51 Y. Zhang, P. Zhu, G. Li, W. Wang, L. Chen, D. D. Lu, R. Sun, F. Zhou and C. Wong, Nanoscale, 2015, 7, 13775-13783.

52 W. Ma, A. Soroush, T. Van Anh Luong, G. Brennan, M. S. Rahaman, B. Asadishad and N. Tufenkji, Water Res., 2016, 99, 188-199.

53 U.S. Geological Survey, Mineral Commodity Summaries 2016, U.S. Geological Survey, Reston, VA, 2016.

54 M. Ben-Sasson, K. R. Zodrow, Q. Genggeng, Y. Kang, E. P. Giannelis and M. Elimelech, Environ. Sci. Technol., 2014, 48, 384-393.

55 M. Ben-Sasson, X. Lu, S. Nejati, H. Jaramillo and M. Elimelech, Desalination, 2016, 388, 1-8.

56 J. Xu, L. Zhang, X. Gao, H. Bie, Y. Fu and C. Gao, J. Membr. Sci., 2015, 491, 28-36.
57 K. Boussu, B. Van der Bruggen, A. Volodin, J. Snauwaert, C. Van Haesendonck and C. Vandecasteele, J. Colloid Interface Sci., 2005, 286, 632-638.

58 R. Zhou, P.-F. Ren, H.-C. Yang and Z.-K. Xu, J. Membr. Sci., 2014, 466, 18-25.

59 J. Lin, W. Ye, M.-C. Baltaru, Y. P. Tang, N. J. Bernstein, P. Gao, S. Balta, M. Vlad, A. Volodin, A. Sotto, P. Luis, A. L. Zydney and B. Van der Bruggen, J. Membr. Sci., 2016, 514, 217-228.

60 K. Hamada, H. Nonogaki, Y. Fukushima, B. Munkhbat and M. Mitsuishi, Dyes Pigm., 1991, 16, 111-118.

61 P. Prádanos, J. I. Arribas and A. Hernández, J. Membr. Sci., 1995, 99, 1-20.

62 M. J. Hajipour, K. M. Fromm, A. Akbar Ashkarran, D. Jimenez de Aberasturi, I. R. d. Larramendi, T. Rojo, V. Serpooshan, W. J. Parak and M. Mahmoudi, Trends Biotechnol., 2012, 30, 499-511.

63 M. Shi, H. S. Kwon, Z. Peng, A. Elder and H. Yang, ACS Nano, 2012, 6, 2157-2164.

64 Y. Chen, Y. Zhang, J. Liu, H. Zhang and K. Wang, Chem. Eng. J., 2012, 210, 298-308. 